\title{
Anhelos (wishes), ideas y el inconsciente freudiano: una conversación con Donald Levy
}

\author{
Carlos de la Puente \\ Universidad de Lima \\ Universidad del Pacífico
}

Recibido: 12 de abril de 2014 / Aprobado: 12 de junio de 2014

En su libro Freud Among the Philosophers, Donald Levy afirma que muchas críticas epistemológicas hechas al psicoanálisis se derivan de un malentendido acerca de lo que Freud llamó "inconsciente”. Para Levy, los anhelos (wishes) y las ideas constituyen lo más importante del inconsciente, y ni anhelos ni ideas tienen un vínculo estrecho con la acción. El autor de este artículo conversó con el profesor Levy sobre este argumento y sobre la posibilidad de que, contrariamente a lo que sostuvo, contenidos mentales vinculados a la acción sean también objeto de la represión.

inconsciente / represión / anhelos

\section{Wishes, ideas and the Freudian unconscious: a conversation with Donald Levy}

In his book, Freud Among the Philosophers, professor Donald Levy challenges the epistemological critiques directed against psychoanalysis on the grounds that those critiques misunderstood what Freud called the unconscious. According to Levy, neither "wishes" nor "Ideas", which are the target of repression, are linked to, or depend upon, action. The author of this article conversed with professor Levy about this defense of psychoanalytic theory and about the opinion, contrary to Levy's, that mental contents linked to action are also part of the Freudian unconscious.

unconscious / repression / wishes

Correo electrónico: CAPUENTE@ulima.edu.pe 
Las relaciones entre el psicoanálisis y la filosofía han sido, por lo general, de tensión y desconfianza mutua. En varios de sus escritos, Freud criticó la dificultad de los filósofos para entender el concepto de inconsciente y para aceptar que la vida anímica, es decir, la vida psicológica, no puede reducirse a la actividad consciente. Mientras que del lado de la filosofía son conocidos los numerables reparos de distintos filósofos a la teoría psicoanalítica. Se ha dicho, por ejemplo, que las nociones de represión y de inconsciente adolecen de oscuridad conceptual (Tugendhat, 1985; MacIntyre, 1958), que las proposiciones psicoanalíticas no pueden ser falseadas (Popper, 1963) y que la teoría psicoanalítica debe recibir confirmación extraclínica (Grünbaum, 1984).

Una postura muy frecuente que los psicoanalistas posteriores a Freud han asumido frente a estos y otros cuestionamientos "epistemológicos" ha sido una que podríamos llamar "fugas hacia adelante". Es decir, han evadido los cuestionamientos $\mathrm{y}$, en vez de responderlos, han reclamado para el psicoanálisis un método de comprobación distinto del que se usa en las otras disciplinas de las ciencias humanas. Hablar, por ejemplo, de una "pluralidad epistémica", o imaginar teorías que no son ni explicativas ni descriptivas, o postular que un interpretacionismo radical, que excluye la idea de causalidad, es el hogar epistemológico del psicoanálisis son algunas de estas respuestas que pueden ser caracterizadas como escapes, es decir, "fugas hacia delante".

No todas las "defensas" del psicoanálisis, sin embargo, han consistido en un movimiento evasivo. Dentro de la filosofía existe ya una tradición de respuestas a las objeciones relacionadas con la capacidad del psicoanálisis de encontrar la verdad, que reconoce que el rigor metodológico es necesario en cualquier intento de conocer el mundo. Estos filósofos afirman que el psicoanálisis, y su método de investigación, que es el encuentro clínico, cumple con estas exigencias. En esta línea, podemos mencionar los artículos de Axel Honneth (2009), de Donald Davidson (1982) y de Marcia Cavell (1993), como ejemplos de esa postura que toma en serio la pregunta sobre el lugar que el psicoanálisis debe ocupar dentro de las ciencias humanas.

En esta misma línea, uno de los esfuerzos más comprensivos por defender la cientificidad del psicoanálisis es el de Donald Levy, en su libro Freud Among the Philosophers. Levy (1996) sabe, por supuesto, que ningún filósofo puede ofrecer hoy una definición indisputable y perfectamente demarcada de lo que se entiende por ciencia, y sabe, además, que la ciencia exige innovación e improvisación y que el método es el sirviente, mas no el guía, de la actividad científica. Con estos dos presupuestos, que por lo demás son aceptados por la mayoría de los filósofos 
de la ciencia, Levy sale al encuentro de cuatro filósofos, quienes, en grados $\mathrm{y}$ en modos distintos, han puesto en duda la consistencia científica de los conceptos psicoanalíticos.

Los cuatro filósofos con los que Levy "polemiza" son Ludwig Wittgenstein, William James, Alasdair MacIntyre y Adolf Grünbaum. A todos ellos, Levy los va a buscar a su terreno, para intentar demostrar que los argumentos con los que cuestionan el valor explicativo de la teoría psicoanalítica están construidos sobre malentendidos.

Como también estoy escribiendo un libro sobre el diálogo entre la filosofía y el psicoanálisis, contacté a este profesor de Filosofía de la Universidad de la Ciudad de Nueva York (CUNY), y durante un año he intercambiado correos electrónicos con él sobre distintos aspectos de su libro. En este artículo presento al lector una parte de nuestra conversación, aquella donde discutimos sobre cuál es el sentido que tuvo para Freud, y el que tiene para el psicoanálisis, el concepto de "deseo" (wish, en inglés). Para Levy, las críticas epistemológicas de MacIntyre y de Grünbaum se derivan de un malentendido acerca de los contenidos del inconsciente. Los contenidos inconscientes son, sostiene Levy, deseos e ideas, y ni los deseos ni las ideas están estrechamente ligados a la acción. Dicho de otro modo, de la conducta observable no verbal de un individuo no se puede inferir cuáles son sus deseos ni sus ideas, menos aún se pueden saber cuáles son sus deseos e ideas inconscientes. Por eso, según Levy, la conducta observable no es el camino para conocer el inconsciente ni para comprobar su influencia en la conducta. Ahora bien, para Levy, que escribe en inglés, hay una diferencia importante entre wishes y desires. Esta es una diferencia que no tenemos en nuestro idioma. No hay traducción al castellano de las obras de Freud que haya optado por anhelo para traducir la palabra inglesa wish (o la palabra alemana Wunsch). Sin embargo, esta diferencia entre wishes y desires es, como se verá en lo que sigue, la clave para entender el argumento de Levy.

Esta no es una "entrevista periodística". Es, más bien, el tipo de entrevista cuya presencia no es infrecuente en las revistas y en los libros de filosofía. Son entrevistas que le dan la oportunidad a un autor de continuar elaborando sus ideas. Eso es lo que creo que ha conseguido esta conversación.

Me parece que para todos los lectores resultará evidente que lo que motiva este intercambio de opiniones es mi admiración por Freud Among the Philosophers.

\section{El "ATAQUE" al PSICOANÁLISIS}

En su libro The Unconscious (1958 [2004]), el filósofo Alasdair MacIntyre sostiene que uno de los logros más significativos de Freud fue el de re- 
describir las conductas de los seres humanos de un modo brillante, como lo hacen los novelistas que descubren aspectos del comportamiento humano hasta ese momento desconocidos. Gracias a esas iluminadas re-descripciones, pudo Freud, de acuerdo con MacIntyre, ensanchar nuestro entendimiento de la mente. Es decir, gracias a que Freud proclamó que había conductas guiadas por intenciones inconscientes, hoy entendemos mejor lo que son las intenciones. El psicoanálisis nos ha permitido, a través del estudio de lo anormal, conocer mejor lo normal. El problema que ve MacIntyre con la teoría psicoanalítica es que no tiene poder explicativo. No entiende MacIntyre cuál es el rol explicativo que tiene el concepto más importante del psicoanálisis: "el inconsciente". Es un mérito indudable de Freud, piensa MacIntyre, el haber llamado la atención sobre las correlaciones que existen entre las experiencias infantiles y la personalidad adulta. Pero esas relaciones causales pueden explicarse sin esa entidad que Freud llamó "lo inconsciente". Sin él podemos comprobar, por ejemplo, que la privación afectiva en los primeros años de vida puede ser la causa de una personalidad dependiente. En otras palabras, de acuerdo con MacIntyre, una concepción prefreudiana de la conducta pudo establecer ya estas relaciones causales. La tesis de MacIntyre, sostenida antes por Wittgenstein, es:
Mi tesis es, entonces, que cuando Freud usa el concepto de "el inconsciente" como un concepto explicativo, fracasa, si no para justificarlo, por lo menos para hacer clara esta justificación. Él [Freud] nos da explicaciones causales, ciertamente, pero estas explicaciones causales pueden y aparentemente deben sostenerse solas, sin referencia al concepto de inconsciente. Él tiene un concepto legítimo de actividad mental inconsciente, ciertamente, pero lo usa para describir comportamiento, no para explicarlo (MacIntyre, 1958, p. 97, mi traducción).

Inconsciente es, por lo tanto, una palabra útil para describir comportamientos, pero oscura si se usa como una entidad teórica necesaria para explicar las relaciones causales sobre las que el mismo Freud llamó la atención.

Adolf Grünbaum, por su lado, ha hecho una crítica al psicoanálisis, aunque más severa, emparentada con la de MacIntyre. Piensa Grünbaum que autores posteriores a Freud, empujados por una actitud paternalista - como Habermas y Ricoeur - han inventado un psicoanálisis "hermenéutico", cuando lo cierto es que la disciplina creada por Freud es científica, basada en hipótesis sobre relaciones causales, y que la verdad de las teorías psicoanalíticas debe probarse acorde con esto, es decir, en pruebas extraclínicas en poblaciones tratadas psicoanalíticamente. 


\section{LA "DEFENSA"}

Hay un punto de la muy variada y creativa defensa de Donald Levy (no se puede resumir en este artículo todos sus brillantes argumentos contra los cuestionamientos de MacIntyre y Grünbaum) en el que me encuentro en discrepancia con él. Este punto es que los contenidos del inconsciente son principalmente anhelos e ideas (y no deseos y creencias), y los anhelos y las ideas son sobre cosas que uno sabe o cree que son imposibles de alcanzar. En un artículo posterior a Freud Among the Philosophers, titulado "Incompleteness and Experimental Unestability in Psicoanálisis" (Estado incompleto e inestabilidad experimental en el psicoanálisis), donde desarrolla con algo más de detalle los argumentos del libro, dice Levy:

[esta idea], voy a argumentar, se deriva de su relevancia en relación a la crítica más importante hecha al psicoanálisis, a saber, la crítica que dice que el psicoanálisis falla cuando es sometido a tratamiento experimental [...]. Brevemente, los contenidos del inconsciente de los que se ocupa el psicoanálisis son ideas $\mathrm{y}$ anhelos inconscientes (ideas and wishes) en contraste con los contenidos inconscientes que son el objeto de la psicología cognitiva (como los deseos y las creencias). Los deseos y las creencias están estrechamente unidos a la acción, mientras que las ideas y los anhelos no. De manera que una gran cantidad de deseos y creencias pueden ser inferidos de la conducta no verbal y de patrones de comportamiento [...] [mientras que] no hay un patrón indicativo de anhelos e ideas (Levy, 2004, p. 180, mi traducción).

\section{Y añade:}

Para entender lo que implica la elección de palabras de Freud, tenemos que recordar que anhelos e ideas comparten en el discurso psicoanalítico peculiaridades que las diferencian de los deseos y las creencias. Así, de acuerdo con Aristóteles, "el anhelo puede ser por lo imposible, e. g., la inmortalidad", mientras que sería raro decir de alguien que desea lo que él sabe o cree imposible de conseguir. La razón de esto es que deseos y creencias están estrechamente unidas a la acción, mientras que anhelos e ideas no [...]. Así, una amplia clase de deseos y creencias puede ser inferida de la acción no verbal [...] (Levy, 2004, p. 180, mi traducción).

Para Levy, entonces, el inconsciente está constituido por ideas y anhelos (ideas and wishes). Ideas y anhelos no están estrechamente vinculados a la acción, porque son, o suelen ser, sobre cosas que la persona cree o sabe que no puede alcanzar. Los anhelos pueden ser sobre lo imposible, mientras que los deseos son, por lo general, de cosas que la persona sabe o cree que puede alcanzar. Por lo tanto, los anhelos y las ideas 
no pueden ser inferidos observando la conducta no verbal de una persona, mientras que los deseos y las creencias, que sí se expresan en la acción, sí se infieren de la conducta no verbal. Ya que este es el caso, puesto que los anhelos y las ideas no tienen una relación estrecha con la acción (i. e., con la conducta observable), porque son sobre cosas que la persona cree o sabe que no puede alcanzar, no es por lo tanto correcto demandar pruebas "observables" del inconsciente. El inconsciente no se despliega en la conducta visible.

¿Si no los "vemos" en las conductas, se pregunta Levy, cómo sabemos de la existencia de anhelos e ideas? Solo a través de la autoatribución, es decir, solo cuando una persona dice yo anhelo tal cosa o yo tengo tal idea. ¿Y los anhelos e ideas inconscientes, que son, según Levy, la esencia del inconsciente? Levy piensa que solo a través de la asociación libre y la observación de la resistencia es que paciente y analista pueden contactarse con lo reprimido, vale decir, con anhelos e ideas inconscientes.

Lo que se reprime es, entonces, de acuerdo con Levy, los anhelos (wishes, en inglés) y las ideas. Las dos preguntas que le hice al profesor Levy, con la que empezamos nuestra polémica son: 1) ¿solo los anhelos se reprimen? y 2) ¿están las ideas separadas de la acción como usted dice? Sobre estas dos preguntas trató parte del diálogo que presento a continuación.

\section{"LA POLÉMICA"}

Carlos de la Puente: Estimado profesor Levy, su libro y su artículo son una poderosa defensa del psicoanálisis. Sobre esta defensa, hay dos aspectos que me han suscitado interrogantes.

En su crítica a MacIntyre, pareciera que su contención es que los anhelos, y nada más que los anhelos, son objeto de la represión. Asumiendo, como usted lo hace, que hay una distinción categórica entre anhelos y deseos, ¿no es el caso que los deseos también puedan ser reprimidos? Además, ¿qué tan cierta es la caracterización de los anhelos (wishes) que usted hace? ¿El anhelo es siempre anhelo de lo inalcanzable? Es probable que le haga estas preguntas porque en castellano no tenemos una diferencia tan categórica entre anhelo y deseo, pero creo que Freud no afirmó que solo los deseos sobre inalcanzables -es decir, los anhelos- son los objetos de la represión.

Donald Levy: En primer lugar, muchas gracias por sus preguntas, que me han obligado a repensar lo que he publicado.

¿Se pueden reprimir los deseos? Cualquiera que diga "no" categóricamente debe sentirse nervioso, como me siento yo, por la naturaleza aparentemente a priori de tal afirmación. Definitivamente, suspendería o retiraría cualquier afirmación que haya hecho que aborde esa negación categórica, si se puede demostrar que Freud ha discutido los deseos reprimidos o si se 
pueden describir casos verosímiles que los involucren. El problema es que los deseos, querer algo, y las necesidades parecen estar estrechamente relacionados, junto con las creencias, a la acción [mientras que los anhelos no]. Esa conexión es tan fuerte que si nos dan dos de los tres [el deseo, la creencia y la acción], muy a menudo podemos inferir el tercero con confianza. Ahora, un deseo reprimido sería presuntamente un deseo inconsciente. ¿Pero qué más se añade al decir que el deseo es reprimido? ¿Eso quiere decir que el deseo no es llevado a cabo? El "deseo reprimido" comienza a parecer un oxímoron. Por supuesto, hay "deseos sublimados", así como deseos reprimidos, pero estos parecen estar marcados por un comportamiento que indica dolor, placer o lucha; es decir, aprobación o desaprobación consciente por parte del sujeto sobre el hecho de evitar actuar con base en el deseo. Me doy cuenta de que aquí existe el peligro de tratar como una verdad profunda lo que solo es un artificio de la forma de pensar de una persona. Sin embargo, sí me parece que ha sido la manera de pensar de Freud, lo cual es importante, ya que aparte de llegar a un marco psicológico que es válido, también estoy interesado en tratar de construir un marco que refleje con precisión el pensamiento de Freud. Cabe mencionar que, cuando escribí el libro, distinguí los deseos de los anhelos basándome únicamente en mis propias intuiciones lingüísticas, las cuales me parecieron ser las mismas que las de Freud. Más adelante, descubrí la larga tradición de hacer la misma distinción que se remonta a Aristóteles, la cual describo con algo más de detalle en un artículo posterior, "Incompleteness and Experimental Untestability in Psychoanalysis" (Estado incompleto e inestabilidad experimental en el psicoanálisis), en Psychoanalysis at the Limit. Epistemology, Mind and the Question of Science (State University of New York Press, 2004). Estoy un poco sorprendido por su afirmación de que "en español no tenemos dos palabras tan diferentes para anhelo y deseo". Nunca he estudiado español, por lo que recurrí al motor de búsqueda Bing, que parece indicar que los verbos desear y querer funcionan en algunos contextos, como lo hacen en inglés los términos wish ("anhelo") y desire ("deseo"). Permítame concluir esta nota diciendo que la dependencia de Freud de la libre asociación podría haber apoyado su idea de una clara distinción entre anhelos y deseos, ya que la técnica implica suspender una acción: no solo el movimiento corporal, sino también la actividad mental, como, por ejemplo, tratar de resolver problemas prácticos o rompecabezas intelectuales.

Carlos de la Puente: Me parece que anhelar (wishing) y desear (wanting, or willing, or intending) son difíciles de separar categóricamente. Quizá es el caso que la voluntad (volition) en los niños se parece más al anhelar, y luego, a través de la experiencia y el aprendiza- 
je, las voliciones se convierten en lo que usted llama deseos, es decir, aquellas voliciones más vinculadas a la acción. La diferencia entre lo que es posible e imposible de alcanzar se instaura paulatinamente en la vida. Por lo tanto, las represiones durante la infancia se dan cuando el niño no diferencia, o está aprendiendo a diferenciar, lo posible de lo imposible. Quizá por eso tiene sentido, y es interesante, diferenciar anhelar de desear y plantear la hipótesis que usted plantea, que la materia de la que está hecho mayormente el inconsciente es de los anhelos, y no de los deseos.

Donald Levy: Claramente la diferencia entre desear y anhelar necesita más trabajo. Sus ideas son muy sugestivas. Se me ocurren dos cosas como extensión de sus comentarios, especialmente aquellos relacionados con las voliciones de los niños. 1) Creo que es extremo decir que el anhelar es siempre sobre lo que se sabe o se cree imposible. Pero sospecho que anhelar siempre (o casi siempre) implica un obstáculo percibido como insalvable, lo que no ocurre con el desear. El obstáculo no tiene necesariamente que ser percibido como insalvable o insuperable, pero tiene que ser un obstáculo que debe ser superado. Si es que estoy en la boletería de un teatro es natural decir: "Quiero (deseo) dos entradas para la función de esta noche", ya que se supone están a la venta y solo necesito pagar. Si todo lo demás es igual, sería extraño decir en esta situación: "Anhelo dos entradas para esta noche". 2) Creo que puedo demostrar la fuerte conexión entre el anhelar y lo imposible (o lo que es muy difícil) citando el contexto en que la palabra represión aparece por primera vez en los escritos de Freud. Freud y Breuer escriben sobre las cosas que el paciente anhelaba (wish to) olvidar, y por eso intencionalmente las reprimió de su pensamiento consciente y las inhibió y suprimió (Standard Edition, vol. 2). Aquí, la represión es vista en sí misma como imposible, o casi imposible. Esto es, tratar de olvidar es una tarea inmensa, por decir lo menos.

Carlos de la Puente: Acerca de los anhelos y las ideas, creo que tenemos dos problemas, profesor. El primero es la posición de Freud sobre estos dos conceptos. Es decir, si es que Freud realmente los vio como alejados de la acción, como usted dice, y si es que estos son los contenidos del inconsciente que predominan. El segundo problema no tiene que ver con Freud y se refiere a la pregunta: “ ¿Es razonable pensar que los anhelos y las ideas se refieren a aspectos de la mente no tan estrechamente vinculados a la acción, independientemente de si Freud los vio así?". Respecto de lo primero, no me parece que Freud haya creído que los anhelos están separados de la acción. Si este fuera el caso, es decir, si fuera cierto que Freud los concibió como distantes de la acción y la conducta visible, la noción de "cumplimiento de deseo" (wish-fulfillment) sería un absurdo, si 
es que no un oxímoron. Cuando Freud habla en La interpretación acerca de los sueños como cumplimientos de un deseo (y, en efecto, Freud usa en esta obra la palabra Wunsch), indica que en este texto él concibe a los anhelos, o cualquiera que sea el nombre de aquello que se expresa y se gratifica en el sueño, como propósitos que el soñante no percibe como imposibles. $\mathrm{Su}$ discusión en La interpretación no deja dudas de que él usa una concepción teleológica de los anhelos. En las traducciones al inglés y al castellano, la frase de Freud referida a los anhelos y a lo que se reprime se lee como "pensamientos con catexias intencionales" (thoughts with a purposive cathexia). Más aún, cuando elabora acerca del primer desear (o el primer anhelo, si consideramos que anhelo es la traducción de Wunsch) en la vida de las personas, se refiere a ellos como la representación de una acción pasada exitosa. "El primer desear parece ser la alucinación catexizada de la memoria de satisfacción" (Freud, 1900). Por lo tanto, la esencia del anhelar es el recuerdo de una acción satisfactoria. Ahora bien, es cierto que en el diccionario de psicoanálisis dice que Freud quería, a través de Wunsch, algo más cercano a la añoranza. Las otras opciones en alemán, Begierde o Lust, no le daban esa posibilidad.

Tampoco es del todo evidente que en el uso cotidiano de la palabra anhelar (to wish, en inglés) se haga siempre alusión a lo que se cree o sabe difícil o imposible de conseguir. "I wish you were here" ("Anhelo que estés aquí") puede ser perfectamente entendido como un pedido de alguien que quiere que yo vaya a verla, cuando, tanto esa persona como yo, sabemos que es posible que yo vaya.

Donald Levy: Usted dice que el cumplimiento del anhelo sería un oxímoron si los anhelos son considerados como separados de la acción, y agrega como evidencia el hecho de que Freud afirma que los sueños son el cumplimiento de los anhelos, razón por la cual él piensa claramente en los anhelos como propósitos que pueden lograrse. Yo le contestaría que, incluso cuando los anhelos son "propósitos que pueden lograrse", no se deduce que [a] el hablante pueda cumplirlos, o que [b] el hablante perciba que puede lograrlos por sí mismo. Un preso en una celda de la cárcel podría anhelar encontrar alguna manera de escapar, pero puede ser que no haya manera de que sea capaz de llevarla a cabo, aunque, por supuesto, si la entrada a un túnel cavada por un prisionero anterior existe, sin que él lo sepa, bajo el suelo de su celda, hay una manera de que él se escape, pero [b] se incumple, ya que él no percibe que exista la posibilidad. $\mathrm{Y}$, por supuesto, si gente poderosa que se encuentra fuera de la cárcel ataca la prisión, él será liberado, pero no por sus propias acciones, por lo que [a] se incumple. Si imaginamos un preso que planea un escape, al reclutar aliados en 
el exterior para sobornar a los guardias $\mathrm{y}$ alquilar un helicóptero para sacarlo, y da las órdenes para hacer lo que sea necesario y promete recompensas a aquellos que él ha contratado, sería extraño decir que él anhela poder escapar. Él sabe, o cree, que puede escapar de esa manera, y está haciendo lo que sea necesario para hacer realidad su deseo de escapar. Otros presos podrían decir de él que quiere escapar; sería extraño que ellos dijeran que [c] "él anhela poder escapar" si saben cuáles son sus planes, y saben que son realistas; [decir [c] podría compararse con que alguien diga: "Me gustaría poder comer el pedazo de pastel que el mesero acaba de colocar frente a mí" cuando no hay impedimentos para hacerlo, ya sea de hecho o que sean percibidos por el hablante]. También sería un tanto peculiar para ellos decir que él "anhela escapar", que suena como lo que una persona excesivamente cortés podría decir, como una manera de señalar al oyente que podría haber algún obstáculo para escapar que es desconocido por el hablante. Esta discusión podría parecer un ejercicio de la filosofía del lenguaje ordinario y nada más, pero si se está de acuerdo en que nosotros, de hecho, hablamos de esta manera, la pregunta sigue siendo: ¿por qué lo hacemos? ¿Qué punto o distinción estamos haciendo al hablar de esta manera? Usted continúa diciendo que "el núcleo de un anhelo es el recuerdo de una acción exitosa", y sustenta esto citando a Freud en el sentido de que el primer deseo "parece haber sido una inversión de energía emocional alucinatoria de la memoria de la satisfacción”. [¿Me podría proporcionar la referencia para esta cita?]. Pero un anhelo puede ser satisfecho sin el accionar de la persona que desea. Si anhelara tener un millón de dólares, mi deseo podría cumplirse gracias a un pariente perdido hace mucho tiempo que me haya dejado ese monto en su testamento. Usted dice que Freud utiliza Wunsch* en lugar de Begierde* o Lust* porque quería una palabra más cercana a la añoranza que a la codicia o la lujuria. Pero solo "añoramos" algo que no podemos producir fácilmente. Sería absurdo decir que "añoro" leer el periódico The New York Times que está colocado en mi escritorio frente a mí; a menos que, por ejemplo, una serie de constantes interrupciones impidan que lo haga.

Usted dice, finalmente, que dependiendo del contexto, anhelar (to wish) puede también ser usado para referirse a algo que puede ser hecho sin obstáculos a la vista. Dice usted: “'Anhelo que vengas' puede ser entendido como un pedido para que yo vaya, cuando sé que es posible para mí ir. ¡Pero su "cuando" cubre una multitud de casos! Decirle a alguien que está en Nueva York desde California "Anhelo que vengas" tiene sentido. ¿Tiene sentido decirle lo mismo a alguien que nos está hablando desde el intercomunicador? Solo si sabemos que es imposible o muy difícil que esa persona venga. 
Carlos de la Puente: Con relación a su concepto de "idea", no me queda tan claro qué entiende usted por esta palabra. Es obvio que entiende algo distinto a creencia, pero no sé si usted está pensando en un juicio o algo así. En todo caso, en el pasaje del ensayo "La represión" que usted cita, ¿no se refiere Freud a "idea" (Vorstellung) como una representación icónica de una acción $\mathrm{y}$, por lo tanto, al elemento cognitivo de la pulsión? Si yo reprimo impulsos hostiles hacia mi hermano o impulsos sexuales hacia mi madre, lo que se reprime es una "idea" en el sentido de una especie de descripción — que puede ser visual- de mi acción. Freud no está hablando de ideas teóricas separadas de la acción, si tal cosa existe. Como podría ser el caso de un creyente que "reprime" la idea de la existencia de Dios.

Donald Levy: Usted parece igualar lo que yo he dicho: que las ideas, a diferencia de las creencias, están separadas de la acción, con algo que yo no he dicho: que las ideas son ideas teóricas separadas de la acción. Pero no tiene que haber nada de teórico en las ideas, solo por el hecho de que ellas (las ideas) no son concebidas para ser actuadas. Si se me ocurre que el bulto en el bolsillo del chico que ha traído una pizza a mi casa es un revólver escondido, muy probablemente descartaré la idea como poco plausible o quizá ridícula, de modo que no actuaré sobre ella, escondiéndome o tirándole un portazo al repartidor de pizzas. Por el contrario, si realmente pienso que el bulto es un revólver, es muy probable que yo haga algo. Nuevamente, prestar un poco de atención a cómo la gente habla $-\mathrm{y}$ no solo la gente que habla inglés- parece proveer un tipo de evidencia que es dramáticamente necesaria cuando tratamos de pensar filosóficamente acerca de la psicología. Estoy de acuerdo en que, en mi esquema, Freud erigió una fórmula basada en distinciones implícitas en el lenguaje ordinario. $\mathrm{Y}$ hay, por supuesto, aquí más que el lenguaje ordinario, como bien lo supo Wittgenstein. Está todo el problema de la verificación. Normalmente no necesitamos el asentimiento (o la autoadscripción) de la persona para adscribirle, con confianza, deseos o creencias. Dados dos componentes de la tríada acción/deseo/creencias, el tercero se infiere fácilmente. Por el contrario, dados los anhelos y las ideas de las personas, es difícil predecir cómo actuarán. Dados dos elementos de la tríada acción/anhelos/ideas, es difícil inferir el tercero. Un empleado puede tener pensamientos (ideas) homicidas dirigidas contra su jefe, y puede anhelar que su jefe esté muerto sin actuar para realizar estas fantasías.

Carlos de la Puente: Creo que es verdad lo que usted dice, en el sentido de que la palabra anhelo parece ser la única que tenemos para referirnos a aquellos propósitos o intenciones que la persona sabe imposibles, o cree que no puede realizar. Pero no creo que el anhelo sea solo eso, y no creo que Freud 
haya concebido el anhelo de esa manera. Nuevamente en La interpretación de los sueños, el llamado sueño paradigmático de Irma parece estar lleno de deseos - anhelos- que Freud podía realizar. Decirle a su amigo Otto que a él (a Freud) le gustaba más M. Ese parece ser un anhelo que no tiene nada de imposible. Lo que separa el anhelo de una persona de su realización en un acto no es la imposibilidad, sino la censura.

Donald Levy: Usted se refiere al anhelo de Freud de decirle a Otto que $\mathrm{M}$ es más querido por él. Y añade que ese anhelo, como varios otros, se refieren a propósitos alcanzables y que es la censura lo que detiene su realización.

Me asombra que usted no considere a la censura como algo que en sí misma produce una forma de imposibilidad. Lo que uno censura en uno mismo se convierte para uno en imposible de decir o siquiera pensar. La censura psíquica deja de parecerse aquí a la censura política o legal. Después de todo, no es una verdad moral universalmente reconocida que uno no debe decirle a los amigos que a uno le gustan más otros amigos. El objetivo que Freud no debería alcanzar aquí es uno que Freud mismo piensa que no debería alcanzar en esta circunstancia especifica. Freud no se puede permitir decirle a Otto: "M me gusta más que tú". Si no se lo puede permitir, es imposible para él decirlo. Es imposible para él en esa situación particular. Si pensamos en lo que dice Freud en abstracto, al margen de cual- quier individuo y su situación particular, por supuesto que es posible para cualquiera decir esas palabras en cualquier momento, pero eso es reducir toda "posibilidad" a una mera posibilidad lógica, lo cual es crudo y trivial al mismo tiempo. Si el tema de uno es la psicología de los seres humanos, lo que es crucialmente relevante es lo que el individuo cree posible o permisible. Después de todo, por favor, considere que, incluso en el sueño, Freud no le dice a Otto: "Me gusta M más que tú". Todo el sueño está hecho para evitar que Freud diga eso, incluso en el sueño. Freud no se puede permitir decir eso ni siquiera en el sueño. Solo después, despierto y después de mucho análisis, él infiere que decir eso es lo que él anhelaba y lo que el sueño le permitió fue expresarlo sin realmente decirlo.

Carlos de la Puente: Muchas gracias, profesor Levy. Me gustaría publicar fragmentos de este diálogo, a la vez que presentar su libro al público hispanohablante.

Donald Levy: Por supuesto, gracias a usted. Quiero decirle que sigo repensando aspectos de mi teoría gracias, en parte, a esta conversación.

\section{CONCLUSIÓN}

El libro Freud Among the Philosophers y el artículo posterior del profesor Levy constituyen esfuerzos importantes por responder a los conocidos cuestionamientos sobre el criterio de verdad que 
se usa en el psicoanálisis y por elaborar esta respuesta, no con la jerga - a veces de aspecto esotérico- que usan los psicoanalistas, sino con el lenguaje que usan los filósofos del lenguaje y de la mente. Dos conclusiones emergen de este esfuerzo.

Primero, para Levy, la perspectiva de la primera persona, es decir, lo que una persona dice acerca de los contenidos de su propia mente, es una perspectiva irreducible e imposible de traducir, y, por lo tanto, un elemento clave en la explicación de la mente y la conducta. $\mathrm{Y}$ esta perspectiva de la primera persona, o de la autoatribución, es el principal criterio de verdad en el psicoanálisis. Para decidir si una interpretación a un paciente es cierta o no, un criterio ineludible es el asentimiento del paciente. Y la teoría de Donald Levy es una sofisticación de los argumentos en favor de la autoatribución como criterio de verdad en psicología, particularmente por una psicología que se interesa por la afectividad, las intenciones y los sueños.

La otra conclusión tiene que ver con la vieja pregunta sobre el modo de cómo debemos interrogar al inconsciente. No es una pregunta de respuesta rápida. Para Levy, es solo a través de la asociación libre y de la resistencia que el inconsciente es "observable" - en un sentido de la palabra observable que él desarrolla-. En el pragmatismo y en la filosofía analítica de la mente, desde los escritos de John Dewey y de Ludwig
Wittgenstein, hay una tradición muy fuerte de considerar lo mental como un derivado de la acción. Pensar, creer, desear o sentir no serían sino tipos de comportamiento o hechos de la vida que no pueden ser entendidos si son desligados de la acción. El psicoanálisis ha resistido siempre esta caracterización de la vida mental, y esta resistencia es la que le ha valido ser etiquetado como una teoría que reproduce los errores del cartesianismo. Pero la intuición de que lo mental no es un derivado de la acción jamás fue abandonada por los psicoanalistas. Lo que hace el profesor Levy es elaborar, con el rigor de un filósofo analítico, esa intuición que tenemos todos los que hemos conducido el psicoanálisis de un paciente: que lo inconsciente, o los contenidos del inconsciente, parecen situarse a medio camino entre las palabras y las acciones.

La obra de Levy es un muy buen primer paso en el camino que debe seguir el psicoanálisis, que es el de utilizar un lenguaje compartido con otras ciencias humanas.

\section{RefERENCIAS}

Cavell, M. (1993). The psychoanalytic mind. Cambridge: Harvard University Press.

Davidson, D. (1982). "The paradoxes of irrationality". En Philosophical essays on Freud (289-305) Cambridge: Cambridge University Press. 
Freud, S. (1900). La interpretación de los sueños. En Freud, S., Obras completas (tomos IV y V).

Grünbaum, A. (1984). The foundations of psychoanalysis. Berkeley, CA: University of California Press.

Honneth, A. (2009). La apropiación de la libertad. La concepción freudiana de la relación del individuo consigo mismo. En Patologías de la razón. Buenos Aires: Katz Editores.

Levy, D. (1996). Freud among the philosophers. New Haven, CT: Yale University Press.
Levy, D. (2004). Incompleteness and Experimental Untestability in Psychoanlysis. En Psychoanalysis at the Limit. Nueva York: State University of New York Press.

MacIntyre, A. (2004). The unconscious. A conceptual analysis. Nueva York: Routledge.

Popper, K. (1963). Conjectures and refutations. Nueva York: Routledge.

Tugendhat, E. (1985). Self-consciousness and self-determination. Cambridge, MA: MIT Press. 